\title{
Pour une typologie des lexèmes construits : entre composition, composition néoclassique et affixation
}

\author{
*Lasserre, Marine \& **Montermini, Fabio \\ UMR 5263 CLLE-ERSS, CNRS \& Université de Toulouse \\ *marine.lasserre@univ-tlse2.fr \&**fabio.montermini@univ-tlse2.fr
}

\section{Introduction}

Surtout depuis le XVII ${ }^{\text {ème }}$ siècle, le français, comme d'autres langues européennes, a emprunté massivement des constituants d'origine grecque ou latine. D'abord utilisés dans le vocabulaire scientifique, ces constituants se retrouvent aujourd'hui dans de nombreuses constructions de la langue courante. Dans les langues classiques, ils apparaissaient pour la plupart dans des composés dans lesquels ils avaient le statut de lexèmes, et certains de ces composés ont d'ailleurs été empruntés dans leur ensemble (par exemple démocratie). Cette notion d'élément de composition prend aujourd'hui une autre dimension, faisant appel à la connaissance étymologique du locuteur. Si l'on nous apprend à l'école que la démocratie est 'le pouvoir du peuple', cette connaissance est-elle toujours mobilisée en synchronie ? Doit-on avoir fait des études classiques pour créer ou comprendre un mot comme footocratie ? Si, comme nous le pensons, la réponse est non, ces éléments doivent-ils, dans les langues actuelles, être toujours analysés comme des éléments de composition? Dans les années récentes, plusieurs travaux se sont penchés sur la composition néoclassique en général ou sur des procédés en particulier, en français et dans d'autres langues ${ }^{1}$. Cependant, une taxinomie statisfaisante qui incluerait élément de composition, élément de composition néoclassique et affixe fait actuellement défaut. Les dictionnaires eux-mêmes peinent à s'accorder sur le statut à donner à ces éléments. L'élément -logie est ainsi désigné comme «élément formant» dans le $T L F i$ et comme «élément entrant dans la composition de... » dans le Grand Robert. Cependant, dans ce dernier dictionnaire, la dénomination de «suffixe » est également employée dans la même entrée, qui donne en outre plus bas la liste des «composés en -logie ». L'entrée -cratie quant à elle n'est que désignée par la tournure « élément entrant dans la composition de » dans le Grand Robert, et n'apparaît pas dans le TLFi. Dans cet article, nous proposons une première taxinomie de ces éléments, en nous concentrant sur ceux qui occupent la partie droite d'un lexème construit et qui, dans la littérature, sont tantôt classés comme éléments de composition, éléments de composition néoclassique ou affixes. Nous proposons, en particulier, une classification fondée sur la notion de construction, telle qu'elle est définie, par exemple, au sein de la morphologie constructionnelle (cf. Booij, 2010). Dans ce cadre, les procédés morphologiques sont considérés comme des phénomènes de la même nature, qui se distinguent essentiellement sur la base de leur degré des propriétés qu'elles spécifient pour les mots qu'ils construisent. Ainsi, la composition typique est un procédé dans lequel seules les catégories des inputs et des outputs (et éventuellement la position de la tête) sont spécifiées, alors que, dans la dérivation affixale typique, plusieurs contraintes, formelles, catégorielles et sémantiques, pèsent sur les outputs, mais il n'y a pas de différence ni en ce qui concerne la nature de ces propriétés, ni en ce qui concerne la manière dont nous considérons qu'ils émergent dans la compétence morphologique des locuteurs, par des généralisations réalisées à partir du lexique existant. Comme nous le verrons, dans notre classification, plusieurs constructions qui correspondent à celle qui est couramment considérée comme la composition néoclassique seront considérées comme très proches des constructions affixales typiques du français. Aucun argument sémantique ou formel ne permet, en effet, de différencier de manière nette ces deux types de phénomènes, et le critère étymologique (provenance d'un composé ou d'un dérivé affixal latin ou grec) ne saurait suffire, à lui seul, pour les distinguer sur le plan synchronique. Même dans un cadre constructionnel, tel celui dans lequel nous nous plaçons, l'identification d'ensembles de procédés distincts est loin de constituer une question purement 
terminologique. Un aspect qui touche directement la composition néoclassique, en particulier, concerne la forme des thèmes des inputs des procédés constructionnels et l'existence de deux niveaux ( registres ») dans la morphologie, au moins, du français (cf. Roché \& Plénat, 2014). À partir de l'identification par Plénat (2008) d'un thème savant (le «thème L », selon sa terminologie) dans l'espace thématique des lexèmes nominaux du français, plusieurs travaux (cf. Roché, 2010 ; Roché \& Plénat, 2014) ont été consacrés à la place de la strate savante dans le système dérivationnel de cette langue, et en particulier à la sélection ou à la construction des thèmes (pseudo-)savants. Notre impression est que la dynamique des contraintes identifiées par Roché \& Plénat (2014) par rapport à la dérivation suffixale typique fonctionne exactement de la même manière pour un certain nombre des constructions qui seraient traditionnellement considérées comme des composés néoclassiques, en particulier en ce qui concerne l'interaction entre la « contrainte de registre » et les contraintes de fidélité à la forme libre.

En (1), nous présentons quelques exemples de doublets pour des lexèmes construits au moyen des éléments -phobe et -cide qui sélectionnent un thème savant (latin ou grec) ou bien un thème que nous pourrions définir comme 'populaire' (les chiffres se réfèrent au nombre de pages référencées par le moteur de recherche Google le 14 mars 2014).

(1)

$\begin{array}{ll}\text { coulrophobe (10.680) } & \text { clownophobe (1.140) } \\ \text { cuniculiphobe (570) } & \text { lapinophobe (39.359) } \\ \text { ornithophobe (3.268) / aviphobe (163) } & \text { oiseauphobe (98) } \\ \text { arachnicide (6.300) / aranéicide (17) } & \text { araignicide (550) } \\ \text { myrmicide (1.300) } & \text { fourmicide (54.300) } \\ \text { piscicide (6.300) / ichty(o)cide (54) } & \text { poissonicide (91) }\end{array}$

Notre but n'est pas ici de discuter les raisons pour lesquelles un type de thème est sélectionné plutôt qu'un autre. Nous nous limitons à observer que, comme le montrent les données ci-dessus, un procédé de construction savant n'entraîne pas nécessairement la sélection systématique (et parfois même pas majoritaire) d'un thème savant, et qu'un élément d'origine latine ou grecque n'entraîne pas nécessairement la sélection d'un thème qui a la même origine, exactement comme pour la suffixation typique.

Cet article est organisé comme il suit : nous commencerons par un état des lieux des constructions traditionnellement définies comme faisant partie de la composition. Ensuite, dans la section 3, nous discuterons des critères qui sont traditionnellement proposés pour distinguer les éléments de composition néoclassique des autres types d'unités lexicales (lexèmes autonomes et affixes), en discutant de leur pertinence et généralité. Dans la dernière partie, nous inscrirons notre réflexion plus précisément dans le cadre de la morphologie constructionnelle, nous proposerons qu'une taxinomie satisfaisante considère les procédés morphologiques comme des constructions, et que les différences observables entre eux relèvent de différents degrés de spécificité d'une ou plusieurs propriétés de ces constructions.

\section{Les composés du français}

La composition ne peut pas être décrite de façon univoque et incontestable, comme l'ont montré par exemple Lieber \& Štekauer (2009). Notre but n'étant pas ici de traiter de la composition en général mais plutôt des différents types d'éléments finaux donnant lieu à des constructions, nous prendrons comme postulat que la composition est la formation d'un lexème à partir de deux lexèmes (ou plus). En français, comme dans d'autres langues européennes telles que l'anglais, l'italien ou l'espagnol, deux types de composition se dégagent, principalement selon l'origine des constituants : la composition native et la composition néoclassique (souvent appelée aussi savante). 


\subsection{Composés natifs}

En français, la composition native (ou ordinaire) est un processus de formation de lexèmes productif pour les combinaisons catégorielles $\mathrm{NN}_{\mathrm{N}}, \mathrm{AN}_{\mathrm{A}}, \mathrm{VN}_{\mathrm{AN}}$ et $\mathrm{AA}_{\mathrm{A}}$ (Fradin 2009 ; Villoing 2012). Elle est dite native car elle implique comme éléments de base des unités qui sont incontestablement des lexèmes du français. L'ordre des constituants, comme pour les autres langues romanes, est XY, où X fournit ses propriétés sémantico-syntaxiques au composé et $\mathrm{Y}$ est un modifieur de $\mathrm{X}$. Dans les termes traditionnels, hérités de la syntaxe, on dit que $\mathrm{X}$ est la tête du composé, ou que les composés du français ont la tête à gauche. En particulier, parmi les composés $\mathrm{NN}_{\mathrm{N}}$, certaines constructions affichent un élément récurrent. Deux sous-types se dégagent, selon que cet élément se trouve à gauche ou à droite.

\subsubsection{Composés de type pause $X$}

Le premier sous-type de composés $\mathrm{NN}_{\mathrm{N}}$ affiche un élément de gauche récurrent. Cet élément est la tête du composé, comme pour tout composé natif. Quelques exemples sont donnés en (2-4) :

(2) poisson X: poisson chat, poisson clown, poisson lune

(3) coin X: coin cuisine, coin terrasse, coin repas, coin jeu, coin parents ${ }^{2}$

(4) pause X: pause café, pause cigarette, pause déjeuner

L'élément de gauche (N1) conserve ici le même sens que le lexème autonome correspondant. Cependant, la relation entre les deux lexèmes est opérée différemment d'un point de vue sémantique. En (2), l'élément de droite (N2) désigne une caractéristique saillante du référent du N1 (la couleur, la forme, la taille, etc.), le N2 est donc un modifieur du N1. En (3-4), le N2 a une fonction de complément du N1, indiquant souvent une fonction, un but ou un lieu. Dans la classification proposée par Bisetto \& Scalise (2005), les composés de (2) sont donc des composés attributifs ( " attributive compounds »), ceux de (3-4) sont des composés subordinatifs («subordinative compounds»). Bien que notre but soit ici principalement de nous attacher aux éléments finaux, l'évocation de ce sous-type nous permet de mettre en perspective le type $X$ fleuve.

\subsubsection{Composés de type $X$ fleuve}

Ce deuxième sous-type de $\mathrm{NN}_{\mathrm{N}}$ affiche un $\mathrm{N} 2$ récurrent. Ce n'est donc plus l'élément tête qui est récurrent mais l'élément modifieur comme nous le voyons en (5)
(5) a. X fleuve : roman fleuve, discours fleuve
b. X clé :
moment clé, mot clé
c. X éclair :
visite éclair
d. X marathon :
journée marathon

Ceci a une incidence sur le sens. En effet, seul un trait sémantique saillant du N2 est conservé dans le composé. Noailly (1990) propose d'analyser ces éléments comme des adjectifs, s'appuyant sur cette extension métaphorique comme critère sémantique et proposant des tests syntaxiques comme (6).

(6) Une visite tout à fait éclair (Noailly, $1990: 43$ )

Sur la base du même critère, Amiot \& Van Goethem (2012), cependant, analysent les composés en -clé du français comme des constructions. En effet, ce comportement, la sélection d'un trait sémantique saillant du N2 qu'elles opèrent et la disponibilité de ces constructions pour la création de nouveaux lexèmes construits sont trois facteurs qui, combinés, nous amènent à considérer les exemples (5-7) comme différents de la composition canonique, dans laquelle n'importe quel lexème - pourvu qu'il ait la catégorie ou les propriétés sémantiques adéquates - peut occuper la place d'un N1 ou d'un N2, sans subir de restrictions ou de sélection sémantique de ses traits. Le fait d'avoir un élément spécifié récurrent dans une posi- 
tion précise et avec des propriétés sémantiques constantes éloigne ces cas de la composition canonique. Remarquons cependant que tous les composés natifs ayant un $\mathrm{N} 2$ récurrent n'opèrent pas, ou pas encore, de sélection d'un trait sémantique saillant du N2, comme on le voit en (7) avec le N2 STAR.

(7) X star: $\quad$ acteur star, chef star, pâtissier star, coiffeur star

Tout en ayant un $\mathrm{N} 2$ récurrent, ces exemples sont uniquement des composés de type coordonné (cf. Bisetto \& Scalise, 2005), comme les composés coordonnés typiques (cf. enseignant-chercheur, horloger bijoutier).

\subsection{Composés néoclassiques}

Contrairement aux composés natifs, les composés néoclassiques mettent en jeu un ou plusieurs constituants non natifs, issus du lexique du grec ancien ou du latin. Ces constituants sont nommés différemment dans la littérature : archéoconstituants (cf. Corbin, 2001), formants néoclassiques, affixoïdes ou, dans la littérature en langue anglaise, «combining forms ». Ces constituants étaient pour la plupart des lexèmes autonomes dans les langues d'origine, bien qu'entrant déjà dans des processus de composition. Ils ont été introduits dans les systèmes des langues modernes uniquement comme constituants liés. Villoing (2012) montre que les patrons productifs de la composition néoclassique et ceux de la composition native ne sont pas identiques. Une caractéristique majeure de la composition néoclassique est la position de la tête sémantico-syntaxique. Contrairement aux composés natifs des langues romanes, la fonction de tête est assumée par le constituant de droite. Dal \& Amiot (2008) parlent, à ce propos, d'« ordre néoclassique ». Cependant, cet ordre, s'il est une caractéristique essentielle des composés néoclassiques, n'est pas exclusif à ce type de formation. Comme le font remarquer Dal \& Amiot (2008), on observe cet ordre à chaque fois que la composition met en jeu un constituant qui ne correspond pas à une forme de lexème du français (8-9). Pour expliquer les cas de lexèmes présentant un ordre néoclassique mais dont les deux constituants sont des lexèmes autonomes du français, Amiot et Dal proposent trois hypothèses : (i) ils sont formés selon les mêmes patrons que les composés néoclassiques, (ii) le deuxième constituant est en voie de grammaticalisation, (iii) la voyelle de liaison qu'ils affichent (/i/ ou /o/) appartient au premier constituant, ce qui n'en fait plus un lexème autonome du français.

(8) pétrodollar, eurosceptique

(9) céréaliculture, théâtrothérapie

Encore une fois, dans une perspective de hiérarchisation des constructions, parmi les composés néoclassiques, plusieurs sous-types se dégagent, selon qu'un constituant soit autonome ou pas, et, dans ce cas, selon sa position. Nous allons nous concentrer ici sur les constituants finaux récurrents, en prenant la question de l'autonomie comme critère premier de distinction.

\subsubsection{Le type $X$ thérapie}

Le premier sous-type affiche un N2 autonome.

$$
\begin{aligned}
& \text { (10) a. X thérapie: } \\
& \text { b. X dépendant : } \\
& \text { c. X pôle : } \\
& \text { d. X sphère: } \\
& \text { e. X forme : }
\end{aligned}
$$

$$
\begin{aligned}
& \text { phytothérapie, théâtrothérapie } \\
& \text { caféïnodépendant, cocaïnodépendant } \\
& \text { cancéropôle, technopôle } \\
& \text { blogosphère, twittosphère, dieudosphère ( }<\text { Dieudonné) } \\
& \text { calciforme, anguilliforme, gazéiforme }
\end{aligned}
$$

Ici, le N2 est la tête du composé, tout comme les N1 POISSON ou PAUSE dont nous avons parlé en 2.1.1. Le premier constituant du composé, s'il peut être autonome ou non, est souvent néoclassique. Que ces N2 récurrents soient d'origine grecque ou non, la voyelle de liaison $/ \mathrm{i} /$ ou $/ \mathrm{o} /$ apparaît dans une très large mesure. Selon Amiot et Dal (2008), cette voyelle marque l'ordre néoclassique et indique à l'interlocuteur 
le sens de l'interprétation du composé. Cependant, certains composés ayant le même ordre que les composés néoclassiques et un N2 non natif n'affichent pas cette voyelle si elle n'est pas présente dans la base :

(11)X show : Hollande show, Aubry show, Chirac show

Cependant, sur la Toile, il est possible également d'observer des composés comme ceux en (12), bien que dans une moindre mesure :

(12) Hollandoshow, Chiracoshow

L'analyse sémantique de ces composés ne peut pas se faire de manière unifiée. En effet, certains lexèmes récurrents conservent l'ensemble du sens du lexème autonome correspondant (Ex. DÉPENDANT, SHOW), pour d'autres des nuances peuvent apparaître dans la relation qui relie les deux lexèmes (cf l'analyse de -thérapie par Dal \& Amiot, 2008 et 3.1.1 ci-dessous). En (10d), le composé sélectionne un des sens du lexème autonome (ici, le sens figuré que peut prendre le lexème SPHĖRE, « Domaine limité où se manifestent et s'exercent l'activité, les attributions ou l'influence de quelqu'un », selon la définition du TLFi). Enfin, en (10e) l'interprétation du sens du composé varie en fonction du N1 (voir là encore l'analyse de Dal \& Amiot (2008)).

\subsubsection{Le type Xcratie}

Un autre sous-type se dégage parmi les composés néoclassiques, dans lequel le deuxième constituant est récurrent mais non autonome.

(13) a. Xcratie : blondocratie, consocratie (< consommateur), théocratie

b. Xlogie : bricologie, moustachologie, psychologie

c. Xphage : anthropophage, chronophage, téléphage

e. Xcide : onclicide, raticide, tutsicide

Ce constituant était un lexème autonome en grec ancien ou en latin. Le constituant de gauche est également souvent néoclassique mais l'emploi d'un constituant natif est également très fréquent. Dans ce cas aussi, la voyelle de liaison /i/ ou / / / est très souvent présente, que le constituant de gauche soit d'origine classique ou non. De plus, des contraintes phonologiques pèsent sur le premier élément, notamment des contraintes de taille (cf. 3.1.3 ci-dessous) : un premier élément de deux syllabes est en majorité préféré. D'un point de vue sémantique, des lexèmes formés avec le même élément final non autonome peuvent recevoir plusieurs interprétations différentes, avec une gamme de sens pour chaque type de composé qui ne sont pas forcément liées synchroniquement. En (14a), par exemple, le sens original du lexème grec

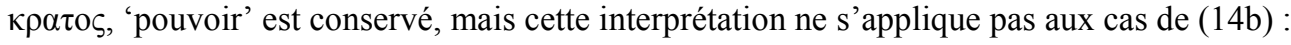

(14) a. Xcratie 'pouvoir de X' : démocratie, argencratie, consocratie

b. Xcratie 'groupe fermé de X s'autovalorisant' : aristocratie, cathocratie, bobocratie

Concernant la disponibilité de ces modes de formation, certains patrons semblent particulièrement actifs, ce qui transparaît dans le très grand nombre de formations plus ou moins occasionnelles recensées sur la Toile. Les différentes caractéristiques que nous venons d'évoquer posent le problème de la nature de cet élément final récurrent et non autonome, et donc du mot construit dans son ensemble. Cette question est également soulevée par les liens qui peuvent exister entre des mots construits selon le type Xthérapie que nous avons décrit en 2.2.1 et le type Xcratie dont il est question ici.

\subsubsection{Autonome vs. non autonome : une frontière floue ?}

Un lexème construit selon un patron néoclassique, mais avec un élément récurrent autonome peut faire partie du même réseau lexical qu'un lexème construit selon le même modèle dont l'élément récurrent non autonome. Là encore, plusieurs cas de figure se présentent. 
(15) a. rizicole ( $<$ riz) 'où l'on cultive du riz, qui concerne la culture du riz' $\rightarrow$ riziculture

b. piscicole (<pisci 'poisson') 'relatif ou propre à la reproduction et à l'élevage des poissons' $\rightarrow$ pisciculture

c. limicole ( $<\lim ^{3}$ 'vase') 'qui vit dans la vase' $\rightarrow *$ limiculture

Comme le soulignent Dal \& Amiot (2008), le lexème CULTURE a le même sens quand il est le deuxième élément de mots construits et quand il est autonome. Cela se vérifie en (15a-b), bien que le sens 'élevage' (15b) ne soit pas particulièrement saillant lorsque CULTURE est autonome. Cependant, à chaque lexème Xcole ne correspond pas forcément un lexème Xculture, Xcole déployant un éventail de sens plus important que Xculture (15c).

Contrairement à CULTURE, le lexème PHOBIE n'a pas le même sens quand il est autonome ou en deuxième position d'un mot construit, ou du moins pas uniquement :

(16) Xphobie
a. 'peur de' :
agoraphobie, aquaphobie, arachnophobie
b. 'aversion envers' :
théâtrophobie, hippophobie
c. 'hostilité à l'encontre de' : xénophobie, homophobie, francophobie
d. ' attraction chimique' : hydrophobie, oléophobie

Quand il est autonome, PHOBIE ne véhicule que le sens 'peur de' (15a). De plus, à chacun des sens listés en (16) correspond, ou peut correspondre, un lexème en Xphobe, alors que -phobe n'est quant à lui pas autonome. Le constituant -phobie est donc assimilable à un constituant attaché dans les constructions Xphobie, ce qui était déjà le cas en grec ancien, où le constituant nome ${ }^{4}$.

Enfin, un autre cas de figure se présente avec -forme et -morphe, qui sont en compétition directe. Alors que le premier peut être autonome et l'autre non, les deux constructions sont rattachées, dans le lexique, à des adjectifs qui présentent le même élément final, à savoir -morphique.
(17) a. calciforme
$\rightarrow$
calcimorphique
b. anthropomorphe
anthropomorphique

Dans d'autres cas encore, le décalage sémantique entre un lexème autonome et son homologue non autonome semble relever plutôt de la relation entre les deux éléments du mot construit, un fait qui est parfaitement compatible avec l'idée d'analyser les composés néoclassiques en termes de constructions. Dans les mots contenant -thérapie, par exemple, le premier élément indique généralement le moyen principal par lequel un traitement est réalisé (une substance, une technique ou autre : antibiothérapie, vaccinothérapie), jamais celui qui le reçoit ou qui l'administre (hippothérapie ne peut jamais désigner un traitement administré à un cheval) $)^{5}$. Il s'agit de restrictions qui sont très semblables à celles que l'on observe dans les procédés d'affixation classiques.

Tous ces exemples montrent donc que la question de l'autonomie ne peut pas constituer à elle seule un critère distinctif entre plusieurs types de composition, voire entre composition et dérivation. Les ouvrages lexicographiques ne nous aident pas davantage à discriminer les deux emplois de ces éléments : le TLFi, par exemple n'indique aucun lien particulier entre -manie et MANIE, alors qu'il considère -thérapie comme l'équivalent de THERAPIE dans des lexèmes composés et fait dériver PHOBIE d'un « emploi indépendant» de l'élément lié -phobie. Pour tous ces éléments, on pourrait, éventuellement, avancer l'hypothèse de l'homonymie, en considérant que, comme nous l'avons vu, chaque couple d'éléments n'est pas parfaitement superposable du point de vue sémantique. Cependant, les réseaux lexicaux que les mots construits entretiennent avec des éléments autonomes et non autonomes, et leur comportement plus général, suggèrent que la question est plus complexe. 


\section{Affixes vs. éléments de composition néoclassique}

La plupart des travaux qui s'intéressent à la construction morphologique au moyen d'éléments néoclassiques mettent en avant un certain nombre de critères pour distinguer ceux-ci, d'une part, des lexèmes ordinaires de la langue et, de l'autre, des affixes canoniques. À quelques exceptions près (par exemple Lüdeling et al. 2002), ces travaux prennent donc le parti plutôt de souligner les différences entre la composition néoclassique et les procédés morphologiques natifs de la langue, et d'identifier une classe délimitée d'éléments de construction néoclassiques, qui peut, éventuellement, se présenter comme l'un des pôles d'un continuum. À partir de ces travaux ${ }^{6}$, il est donc possible de lister quelques critères qui serviraient à définir, au moins de manière prototypique, les éléments de construction néoclassiques en les distinguant des autres types d'unités qui interviennent dans les procédés morphologico-constructionnels. Dans ce qui suit nous distinguerons, parmi ces critères, ceux qui concernent les propriétés distributionnelles et combinatoires des éléments, leur sémantique (interne et externe) et leur forme. La liste proposée n'est pas exhaustive : nous nous limitons, en effet aux critères qui sont pertinents pour le français et/ou qui semblent être plus consensuels parmi les auteurs. Dans tous les cas, nous verrons que ces critères sont insuffisants aussi bien pour délimiter une classe homogène d'objets que pour distinguer les éléments qui en feraient partie des autres unités morphologiques. Nous verrons aussi que le critère étymologique (origine grecque ou latine des éléments et des composés en question), est indépendant des autres, et au final, comme il était prévisible, n’a pas de pertinence pour une analyse purement synchronique des données.

\subsubsection{Critères distributionnels}

Le premier ensemble de critères que nous considérons concerne les propriétés distributionnelles et la combinabilité des éléments qui interviennent dans les procédés de morphologie constructionnelle. Le caractère lié des éléments de composition néoclassique, que nous avons traité en 2.2.3, les rapprocherait plutôt des affixes canoniques, mais plusieurs autres propriétés les distingueraient de ceux-ci. Parmi cellesci les plus souvent citées sont la possibilité d'occuper indifféremment la première ou la deuxième position dans un mot complexe (alors qu'un affixe occupe toujours la même position dans un mot complexe, par exemple, en français, initiale ou finale) et la possibilité d'être à leur tour dérivés par affixation (alors qu'un affixe ne peut généralement pas se lier à un autre affixe).

En ce qui concerne la position occupée dans le mot complexe, les auteurs se basent souvent sur des couples d'exemples comme graphologue / logographe, cinéphile / philosophe pour souligner la distance entre ces éléments et les affixes canoniques qui ne peuvent occuper que la position la plus à gauche ou la plus à droite dans un mot complexe. En réalité, cette 'mobilité' des éléments de composition néoclassique nous semble devoir être largement redimensionnée. Même dans le cas de ceux qui présentent cette possibilité on observe une spécialisation en tant qu'élément initial ou final, en particulier dans les nouvelles formations, et lorsque ces éléments sont liés à des lexèmes autonomes de la langue courante, ce qui témoigne de leur statut d'exposants de constructions spécifiques. Nous avons extrait du TLFi les lexèmes complexes qui comportent un des cinq éléments -graph-, -log-, -metr-, -morph- et - phil- $^{7}$ en première ou dernière position et, parmi ceux-ci, nous avons distingué ceux qui comportent un lexème non autonome (par exemple logonévrose ou futurologue). Les résultats sont donnés dans le Tableau 1.

\begin{tabular}{lcccc}
\hline élément & $\mathbf{2}^{\mathbf{e}}$ position & $\begin{array}{c}\text { dont avec base } \\
\text { autonome }\end{array}$ & $\mathbf{1}^{\mathbf{e}}$ position & $\begin{array}{c}\text { dont avec base } \\
\text { autonome }\end{array}$ \\
\hline -metr- & 288 & 57 & 7 & 1 \\
\hline -graph- & 196 & 23 & 19 & 8 \\
\hline -log- & 170 & 50 & 28 & 6 \\
\hline -phil- & 123 & 34 & 16 & 2 \\
\hline -morph- & 40 & 3 & 20 & 12 \\
\hline
\end{tabular}

Tableau 1: nombre des mots complexes comportant quelques éléments de composition néoclassique en première ou deuxième position 
Les données ci-dessus doivent, bien sûr, être considérées comme une simple indication des tendances qu'il est possible d'observer avec les éléments en question. Il nous semble clair, cependant, qu'il n'est pas possible d'affirmer qu'il s'agit du même élément qui peut occuper indifféremment la première ou la deuxième position dans un mot composé. Cela apparaît de façon très évidente lorsqu'on observe la sémantique de certains d'entre eux : lorsqu'il est final, -logue construit massivement des noms [+humain] qui désignent des spécialistes (archéologue, futurologue), plus rarement des noms indiquant des types de textes (décalogue). Lorsqu'il est initial, en revanche, il peut indiquer un discours (logomachie) ou, plus souvent, un mot (logosyllabique) ou la faculté de langage en général, surtout dans des mots qui appartiennent au domaine psycho-médical (logopathie), mais le sens de 'connaissance', 'science' est tout à fait exclu $^{8}$. Le seul, parmi les éléments considérés, pour lequel les deux emplois (premier ou deuxième élément d'un composé) seraient comparables est -morph-. Cependant, dans la plupart des usages en tant que premier élément, morph(o)- ne signifie pas simplement 'forme' (comme dans anthropomorphe ou morphogénèse), mais est plutôt une contraction de morphologie, aussi bien dans le sens qu'il a dans le domaine des sciences de la vie et de la terre (morphopsychologie) que dans celui qu'il a en linguistique (morphosyntaxe), un phénomène qui n'est pas sans rappeler le glissement que l'on observe avec des éléments comme auto- ('de soi-même' > 'automobile' autoradio) ou éco- ('maison' > 'écologie', écotaxe).

Concernant la possibilité, pour les éléments de construction néoclassique, de servir à leur tour de bases à des procédés dérivationnels, et en particulier d'être suffixés, on peut formuler des observations très semblables à celles ci-dessus. Dans le Tableau 2 nous indiquons les mots suffixés présents dans le TLFi à partir de chacun des cinq éléments considérés ci-dessus, avec la date de première attestation indiquée dans le dictionnaire.

\begin{tabular}{lllll}
\hline -graph- & -log- & -metr- & -morph- & -phil- \\
\hline graphème (1913) & logique (1245) & métrique (1795) & morphème (1921) & \\
graphique (1757) & & & \\
graphisme (1875) & & & \\
graphite (1801) & & & \\
\hline
\end{tabular}

Tableau 2 : dérivés à partir de quelques éléments de composition néoclassique dans le $T L F i$

La famille morphologique de chacun de ces éléments est extrêmement réduite et, comme on le voit, aucun d'entre eux ne peut être considéré comme une base morphologique disponible en français (aucun n'a donné lieu à des dérivés après la première moitié du $\mathrm{XX}^{\text {eme }}$ siècle $)^{9}$. Si les lexèmes du Tableau 2 sont analysables ou partiellement analysables (et tous ne le sont pas), cela ne confère pas automatiquement à la partie qui précède le suffixe un statut particulier et unique : dans certains cas seul, le suffixe peut être clairement reconnaissable (comme dans logique), dans d'autres cas on pourrait considérer qu'il s'agit du thème alternatif d'un autre lexème (par exemple, on pourrait considérer que MÉTRIQUE est à MESURER ce que SIGNALÉTIQUE est à SIGNALER ou ABANDONNIQUE à ABANDONNER).

\subsubsection{Critères sémantiques}

Du point de vue sémantique, l'argument principal qui est avancé pour distinguer les composés néoclassiques des lexèmes dérivés canoniques, et donc les éléments de composition des affixes, concerne la nature du sens que chacune de ces classes d'éléments véhiculerait : (typiquement) dénotatif ou lexical dans le premier cas, (typiquement) grammatical dans le deuxième. Cette distinction est exprimée de manière claire par Corbin (2001), qui affirme que « les affixes constructionnels sont porteurs d'un sens instructionnel » (Corbin, 2001: 43), alors que «archéoconstituants et fractoconstituants ont un sens de nature descriptive et non instructionnelle » (Corbin, $2001: 44$ ) (cf. aussi Iacobini, $2004: 85$ ). Apparemment, il s'agit d'une distinction très forte et utile pour établir des distinctions nettes entre les éléments. Cependant, dans l'absolu, même s'il est possible d'identifier des tendances, cette distinction n'est pas toujours aussi aisée. Pour reprendre les exemples ci-dessus, nous ne voyons pas quel pourrait être le sens référentiel d'un élément comme -log-, qui, il est vrai, peut renvoyer dans certains cas aux concepts de 'mot' ou 'discours', mais certainement pas dans la plupart des cas où il est postposé à la base, qui - comme nous 
l'avons montré - constituent le seul emploi disponible pour cet élément en français. Comme l'argumentent Amiot \& Dal (2007 : 332-333), ce -logue, qui sert à construire des noms de spécialistes, a le même comportement syntaxique et sémantique d'autres éléments, comme -iste, dont le statut affixal ne fait pas de doute. En fait, il existe un nombre important d'éléments de composition néoclassique, en particulier finaux, qui participent de la dynamique, illustrée par Roché (2011), entre opération sémantique associée à un procédé de construction et « schème de nomination » (Roché, $2011: 21-22)$ : les opérations morphologiques réalisent des manipulations abstraites des traits sémantiques associés à un lexème (par exemple des rôles thématiques), alors qu'un schème de nomination assure, via la référence, le lien entre les objets linguistiques que sont les lexèmes et des classes d'objets du monde. Les relations entre ces deux niveaux peuvent être multiples : si, dans certains cas, un schème de nomination spécifique peut avoir un procédé morphologique associé de façon stable (l'exemple de Roché est celui des noms d'arbres en -ier du français), il peut y avoir des cas de concurrence entre des procédés morphologiques pour le même schème de nomination (par exemple les noms d'instrument en français, qui peuvent être construits par les suffixes -eur, -ier, par conversion, par composition VN, etc. ; cf. Roché, 2011 : 22), et, bien sûr, un même procédé peut servir à plusieurs schèmes de nomination (le suffixe -ier en français ne construit pas uniquement des noms d'arbres, et même pas uniquement des noms). Dans le cas de -logue, si nous acceptons l'idée qu'il s'agit de l'exposant d'un procédé morphologique, on peut considérer qu'il est en concurrence avec d'autres procédés dans la construction de noms de spécialistes à partir d'un nom d'objet ou de concept (par exemple les suffixations en -iste, en -ien, mais aussi, pourquoi pas, -graphe, cf. cartographe, iconographe). Trois arguments au moins nous semblent confirmer cette idée. D'une part, il n'est pas rare d'observer des mots construits au moyen d'éléments de composition néoclassique pour lesquels la charge sémantique de ces derniers est nulle, et tout à fait comparable à celle de ces affixes dont l'unique fonction est celle de modifier la catégorie lexicale des bases auxquelles ils se lient (par exemple les suffixes qui forment des adjectifs de relation, cf. Roché 2009) :

(18) Je fais régulièrement appel à vos connaissances cinéphiles pour pallier les miennes qui restent défaillantes dans bien des domaines du cinéma.

[http://www.effets-speciaux.info/forum/viewtopic.php?p=19207]

Qui plus est, ces prétendus djihadistes sont un ramassis de criminels sortis pour l'occasion des prisons des dictatures pétrolifères wahhabites du golfe persique, Arabie Saoudite et Qatar afin de semer la terreur et la haine chez un voisin honni.

[http://tempsreel.nouvelobs.com/la-revolte-syrienne/20130315.OBS2044/reactions/]

Le tout autour et avec les excellentissimes choix discologiques de sebastien Tison, dont la playlist figure dans le même blog.

[http://patwhite.com/node/4314]

Dans les exemples de (18), aucun des éléments de composition néoclassique qui apparaissent dans CINÉPHILE, PÉTROLIFÈRE ou DISCOLOGIQUE ne peut être considéré comme ayant une quelconque valeur référentielle. Ces adjectifs rentrent en concurrence avec des adjectifs de relation 'purs' (par exemple FILMIQUE ou PÉTROLIER), ou bien peuvent être expliqués sur la base d'autres contraintes, par exemple une contrainte de taille phonologique, selon laquelle un mot dérivé de trois syllabes est préférable à un de deux syllabes, et une contrainte lexicale (le nombre de mots se terminant en -logique sur le total des adjectifs en -ique en français), dans le cas de DISCOLOGIQUE. La concurrence entre procédés de suffixation et composition néoclassique est d'ailleurs avérée dans certains cas. Plusieurs travaux récents, par exemple, ont montré que certains préfixes (par exemple anti-) peuvent construire des adjectifs en prenant comme bases aussi bien des noms que des adjectifs de relation à leur tour construits sur la base d'un nom (cf. en particulier Hathout 2011). Or il n'est pas rare que, dans des séries de mots préfixés qui sont en concurrence, des composés néoclassiques interviennent à côté de suffixés typiques :

(19)

$\begin{array}{llll}\text { ALLERGIE } & \text { anti-allergie } & \text { anti-allergique } & \text { anti-allergène (20a) } \\ \text { CANCER } & \text { anti-cancer } & \text { anti-cancéreux } & \text { anti-cancérigène (20b) }\end{array}$


(20) a. A l'origine c'était un médicament anti allergène, prescrit pour les conjonctivites, rhinites essentiellement et notamment pour les enfants.

[http://m.yabiladi.com/read/5795655/veut-grossir.html]

b. Le but est d'administrer des médicaments anti-cancérigènes par voie intraveineuse afin de réduire la taille de la tumeur.

[http://www.divine.ca/fr/mobile/articles/story.php?newsitemid=2302]

Certes, on pourrait soutenir qu'il existe des différences sémantiques entre les mots de (19) et argumenter qu'un médicament anti-cancérigène ne soigne pas le cancer mais en empêche la surgissance. Toutefois, l'analyse des mots en contexte (en particulier (20b)) invite à plus de prudence dans des conclusions qui assument qu'il existe une isomorphie parfaite entre forme et sens. Enfin, dans les relations que les éléments de composition néoclassique entretiennent entre eux, on peut observer des dynamiques de concurrence entre procédés morphologiques pour le même schème de nomination semblables à celles décrites pour les procédés affixaux canoniques par Roché (2011) rappelées ci-dessus. Considérons, par exemple, des couples comme PSAMMOPHILE / SABULICOLE ('qui vit dans le sable') ou un ensemble comme BiBLIOLÂTRE, BIBLIOMANE, BILIOPHAGE, BIBLIOPHILE ('amateur (compulsif) de livres', 'gros lecteur'). Au delà du sens le plus immédiat (et naï) que nous pouvons attribuer à quelques-uns de ces éléments (par exemple, -phage 'manger' ou -phile 'aimer'), il nous semble que la distinction entre procédés morphologiques et schèmes de nomination, et le fait de prévoir une interface entre les deux, permet de rendre compte de ces exemples mieux qu'une analyse décompositionnelle qui essayerait d'attribuer une signification à chacun des éléments identifiés, même s'il s'agit d'une signification abstraite et indéterminée de laquelle on fait dériver les sens effectivement attestés.

\subsubsection{Critères formels}

Le principal critère phonologique qui est mis en avant pour les composés néoclassiques et qui est pertinent pour le français ${ }^{10}$ est l'apparition, entre les deux éléments, d'une voyelle $/ \mathrm{i} /$ ou $/ \mathrm{o} /$. En général on considère, sur des bases étymologiques, que / $/$ appartient au lexique d'origine grecque et $/ \mathrm{i} /$ à celui d'origine latine. En réalité, ce point déjà peut être mis en discussion. Par exemple, dans un corpus de mots en -vore que nous avons constitué à partir des dictionnaires (TLFi et Grand Robert) et de la Toile ${ }^{11}$, seuls 123 lexèmes, soit $69,89 \%$ du total, affichent la voyelle attendue /i/. Par contre, 40 d'entre eux (22,73\%) affichent la voyelle $/ \mathrm{o} / .28$ d'entre eux $(15,91 \%)$, comme par exemple bouquinovore, sont construits à partir d'un lexème qui ne contient pas de / $/$ /, et l'apparition de cette voyelle n'est donc justifiée ni par un souci de fidélité à la base ni par l'élément de composition lui-même, qui est d'origine latine.

Indépendamment de son timbre, le statut de cette voyelle 'de liaison' peut être discuté. Selon une approche compositionnelle (qui est celle adoptée, plus ou moins explicitement, dans la plupart des travaux de morphologie), on peut considérer que cette voyelle 'appartient' au premier élément, au deuxième ou qu'elle constitue un troisième élément, autonome des deux autres (cf. Bauer 1998). Cette dernière hypothèse peut être adoptée même sans attribuer à la voyelle en question le statut d'un morphème, et donc un sens spécifique, mais en considérant que son rôle est simplement celui de marquer le statut de mot composé de l'ensemble (sur les marqueurs de composition, cf. en particulier Ralli 2007). Il est plus fréquent, cependant, d'intégrer la voyelle au premier élément, d'une part parce que la même voyelle apparaît aussi lorsque le deuxième élément est un mot par ailleurs autonome dans la langue (psychosocial, équidistant), et d'autre part parce que certains lexèmes peuvent aussi subir une modification phonologique qui leur associe la même voyelle lorsqu'ils sont employés en composition, que ce soit avec des éléments néoclassiques ou pas (cf. sociologie vs. socio-professionnel). La présence obligatoire de segments constants, quelle que soit l'analyse que l'on en donne, serait donc un critère qui permettrait de distinguer les composés néoclassiques des dérivés canoniques. En réalité, considérer certains éléments de construction néoclassiques comme simplement des exposants de procédés morphologiques (c'est-à-dire de constructions) nous conduit à les priver du statut d'objets lexicaux et à attribuer leurs propriétés, y compris formelles, à la construction elle-même. De ce point de vue, tant la structure des lexèmes construits que les phénomènes de variation auxquels elle est sujette peuvent recevoir un traitement qui est commun aux dérivés 
canoniques et à certains composés néoclassiques qui leur sont proches. Nous avons évoqué plus haut, par exemple, l'existence de contraintes de taille, pour les composés néoclassiques qui sont probablement très semblables à celles qui valent pour la dérivation canonique (cf. Plénat 2009 ; Plénat 2011 : 157) ; de plus, Plénat (2011 : 156-157), en reprenant des données de Corbin \& Plénat (1992), observe que le fonctionnement de l'haplologie est le même pour les dérivés et certains composés avec un élément néoclassique final. Enfin, nous sommes convaincus que la meilleure manière de caractériser le fonctionnement phonologique d'un procédé morphologique est de considérer, en adoptant une perspective 'output-oriented', qu'il prend la forme d'une série de contraintes sur la structure phonologique du lexème construit. Cela est très clair dans certains cas 'classiques' d'allomorphie suffixale, pour lesquels il est possible d'identifier un défaut et une ou plusieurs variantes moins optimales qui peuvent être sélectionnées, par exemple pour satisfaire des contraintes de fidélité à la base. C'est le cas, par exemple, de -able $>-i b l e>-u b l e$ (cf. Plénat 1988), ou des différentes variantes du suffixe agentif -tion, qui peut apparaître sous différentes formes lesquelles peuvent être hiérarchisées en ordre de préférence ([asjõ] étant la forme par défaut). Concernant les composés néoclassiques, nous considérons que précisément la présence massive des voyelles /i/ ou /o/ détermine une série de contraintes sur la forme des lexèmes construits qui prévoyent que l'exposant à proprement parler soit de préférence précédé de l'une ou l'autre de ces voyelles (liée, mais pas de manière exclusive, à leur origine), à défaut d'une autre voyelle ou, dans de rares cas, d'une consonne. Un cas qui permet de montrer de manière claire ce fonctionnement est celui des lexèmes en -phone construits sur le nom d'une langue et qui en désignent les locuteurs (comme arabophone). À partir de la liste des langues du monde de la Wikipédia française ${ }^{12}$, nous avons obtenu, par des recherches sur la Toile, 170 noms de locuteurs construits par -phone. En (21) nous en donnons la liste en les distinguant selon le segment qui précède la séquence [fon] :

\begin{tabular}{|c|c|c|}
\hline [ofon] & \multicolumn{2}{|c|}{ créolophone, francophone } \\
\hline [afon] & aymaraphone & \\
\hline [ifon] & swahiliphone & \\
\hline \multicolumn{2}{|c|}{ [efon] $] /[\varepsilon$ fon] japonaiphone } & 3 \\
\hline [ufon] & zoulouphone & \\
\hline [Vfon] & hébreuphone & \\
\hline [Cfon] & ocphone & \\
\hline
\end{tabular}

La forme [ofon] fonctionne clairement comme le défaut. Cependant, l'intérêt de ces données est précisément que, dans les cas extrêmes, elles permettent de montrer que cette contrainte sur la forme du mot construit peut être contournée pour en respecter d'autres, par exemple, une contrainte de fidélité à la base, en particulier lorsque celle-ci se termine elle-même par voyelle.

Pour conclure, les critères considérés dans cette partie nous semblent avoir été élaborés sur un ensemble d'objets (les éléments de composition néoclassique) préalablement identifiés sur la base de critères qui n'étaient pas entièrement linguistiques (leur origine latine ou grecque, leur emploi dans des domaines technico-scientifiques) et non sur la base des propriétés individuelles des éléments qui en font partie. S'il est vrai, par exemple, que certains éléments peuvent (rarement) apparaître en première ou en deuxième position dans un mot composé et que d'autres peuvent (rarement) être suffixés, cela n'autorise pas à étendre ces caractéristiques à l'ensemble des éléments que l'on considère comme des membres de cette classe.

\section{Conclusion : une taxinomie des procédés de construction}

Dans les sections précédentes nous avons vu que ni le critère étymologique ni les critères sociohistoriques sont entièrement adéquats pour caractériser une classe de 'composés néoclassiques' qui serait 
uniforme et bien délimitée en synchronie. Au contraire, les mots construits d'origine ou d'inspiration allogène qui ont été introduits en français depuis le XVII ${ }^{\text {ème }}$ siècle, d'abord dans les domaine techniques et scientifiques et ensuite, de plus en plus, dans la langue courante, participent, comme le reste des lexèmes - construits, non construits ou partiellement analysables - à la dynamique du lexique, à la mise en place de réseaux à son intérieur et à l'émergence de modèles que les locuteurs peuvent exploiter pour la création de nouvelles unités. Au lieu de constituer un domaine à part de la dérivation, une sorte de 'comorphologie' constructionnelle, les procédés de formation issus du lexique néoclassique interagissent avec les procédés 'natifs' de la langue à plusieurs niveaux contribuant à façonner un système morphologico-dérivationnel qu'il nous semble plus utile de considérer comme unitaire. En conclusion, nous essayerons, sur la base des observations ci-dessus, d'établir une taxinomie des procédés de construction du français, avec une attention particulière pour ceux où l'élément récurrent occupe la position finale (et dont le prototype est la suffixation), en observant la pertinence pour chacun d'eux d'un certain nombre de paramètres formels, catégoriels et sémantiques. Le cadre théorique dans lequel notre proposition est ancrée est celui de la morphologie constructionnelle (cf. en particulier Booij 2010b); dans ce cadre, les mots complexes ne sont pas dissociés de la règle qui les a produits, mais contribuent à définir des schémas constructionnels abstraits, dans lesquels certaines propriétés peuvent être remplacées par des variables plus ou moins spécifiques. Deux propriétés de l'approche constructionnelle à la morphologie nous semblent particulièrement pertinentes pour notre analyse. En premier lieu, les schémas constructionnels ne constituent pas des ensembles discrets de procédures ou de propriétés définies indépendamment, mais peuvent être mis en relation les uns avec les autres et en particulier être hiérarchisés. Ainsi, pour reprendre un de nos exemples de 2.1.2, les mots en $X$ fleuve peuvent être considérés comme un sous-ensemble des composés $\mathrm{NN}$ avec tête à gauche dans lesquels certaines propriétés du deuxième élément sont spécifiées comme héritées du lexème FLEUVE. À leur tour, les composés NN constituent un sous-ensemble du schéma plus général de composition. En (22) nous proposons une schématisation de cette hiérarchie :<smiles>N#CNNN[W]#N</smiles>

Deuxièmement, et en cohérence avec ce qui vient d'être dit, la morphologie constructionnelle ne nous oblige pas à établir des frontières entre les différents types de procédés et à identifier, donc, plusieurs sous-modules (par exemple un module pour la dérivation affixale et un pour la composition); au contraire, la dérivation affixale et la composition sont vues comme des procédés de la même nature qui présentent, simplement, différents niveaux et différents types de spécifications dans les éléments qui les composent (cf. Booij 2010a, mais aussi Roché 2009).

Pour revenir aux types de constructions qui nous intéressent ici, nous proposons en particulier la classification suivante :

$\begin{array}{llllll}1 & 2 & 3 & 4 & 5 & 6 \\ \text { composés typiques }> & \mathrm{X} \text { fleuve }> & \mathrm{X} \text { dépendant }> & \mathrm{X} \text { thérapie }> & \mathrm{X} \text { Cratie }> & \text { suffixés typiques }\end{array}$

Comme nous l'avons répété, nous ne nous intéressons ici qu'aux constructions où l'élément de droite est spécifié. Il est possible, et même certain, que d'autres types de constructions, parallèles ou dépendantes de celles listées en (23), devraient être identifiées, si l'on s'intéressait également aux éléments de gauche. Par exemple, nous classerions certainement les composés du type pause $X$ en une position intermédiaire entre 1 et 2 , puisqu'ils possèdent un élément (le $\mathrm{N}$ de gauche) qui est spécifié et qui détermine quelques propriétés syntaxiques du composé (par exemple le genre et les traits de sous-catégorisation), à la différence du type $X$ fleuve où l'élément spécifié (celui de droite) est, de ce point de vue, neutre. Parmi les 
propriétés que nous avons considérées pour établir cette classification, une (i) concerne la nature de la construction elle-même, d'autres sont d'ordre lexical (ii-iii), syntaxico-sémantique (iv-v) ou phonologique (vi-vii). Nous les listons ci-dessous et, dans le Tableau 3, nous montrons leur pertinence pour chacun des types de procédés identifiés.

(i) présence d'un élément spécifié : cette propriété ne sert qu'à distinguer les composés typiques, pour lesquels aucun des éléments n'est formellement ou sémantiquement spécifié, des autres types de constructions ;

(ii) caractère lié des éléments : cette propriété se réfère à la possibilité qu'un élément corresponde formellement et sémantiquement à une unité qui est par ailleurs un lexème autonome de la langue (par exemple -phobie / PHOBIE vs. -cratie) ;

(iii) participation à des réseaux lexicaux qui comprennent des éléments non autonomes : cette propriété sert à distinguer les constructions comme $X$ dépendant, qui ne rentrent dans des réseaux lexicaux qu'avec des constructions comprenant un autre lexème autonome (par exemple $X$ dépendance) de celles comme $X$ phobie, qui rentrent dans des réseaux lexicaux avec des constructions comprenant des unités liées (par exemple $X$ phobe);

(iv) spécification sémantique : cette propriété se réfère au fait que, lorsqu'un élément d'une construction a un correspondant autonome, les propriétés sémantiques des deux sont parfaitement superposables (cette propriété n'est pas pertinente pour les types 5 et 6);

(v) héritage des propriétés syntaxico-sémantiques : cette propriétés distingue les constructions (comme la composition typique du français) où les propriétés syntaxico-sémantiques du lexème construit correspondent aux propriétés syntaxico-sémantiques de l'élément de gauche, lorsqu'il peut aussi être autonome, des constructions (comme dans la composition 'allogène') dont les propriétés syntaxicosémantiques correspondent à celles de l'élément de droite (cette propriété n'est pas pertinente pour les types 5 et 6 );

(vi) contraintes sur la structure segmentale : cette propriété se réfère à la sensibilité à de contraintes sur la structure segmentale de la base, qui peuvent comporter la présence préférentielle d'un segment déterminé (/// ou /i/, comme nous l'indiquons dans le tableau) ou la sélection d'un allomorphe 'savant' ou pseudo-savant pour l'élément de gauche ;

(vii) contraintes de taille : cette propriété se réfère à la sensibilité à des contraintes de taille sur la structure prosodique de l'élément de gauche. 


\begin{tabular}{|c|c|c|c|c|c|c|}
\hline & 1 composés & $2 \mathrm{X}$ fleuve & $3 \mathrm{X}$ dépendant & $4 \mathrm{X}$ phobie & 5 Xcratie & 6 suffixés \\
\hline (i) & - & + & + & + & + & + \\
\hline (ii) & - & - & - & - & + & + \\
\hline (iii) & - & - & - & + & + & + \\
\hline (iv) & - & + & + & + & n.p. & n.p. \\
\hline (v) & $\mathrm{g}$ & $\mathrm{g}$ & $\mathrm{d}$ & $\mathrm{d}$ & n.p. & n.p. \\
\hline (vi) & - & - & $+(0)$ & $+(\mathrm{o} / \mathrm{i})$ & $+(\mathrm{o} / \mathrm{i})$ & + \\
\hline (vii) & - & - & + & + & + & + \\
\hline
\end{tabular}

Comme on le voit, la prise en compte de propriétés explicites identifiées en synchronie et qui ne reposent pas sur des considérations socio-historiques nous permet, d'une part, de voir que l'ensemble des 'composés néoclassiques' en français ne constitue pas une classe homogène d'éléments et qui a une pertinence pour la compétence synchronique des locuteurs, et, d'autre part, de mettre en perspective les différents procédés constructionnels d'une langue en les considérant comme des cas particuliers à l'intérieur d'un seul composant, qui est le composant lexico-constructionnel. De plus, l'analyse que nous proposons a mis en lumière le fait que l'observation des procédés de construction ne peut pas faire l'impasse sur des analyses fines de leurs propriétés sémantiques, de leur disponibilité (voire de leur productivité) et de leurs contextes d'emploi. Il s'agit, comme nous l'avons indiqué, d'une première proposition dans une taxinomie plus globale des procédés constructionnels du français, dans une approche constructionnelle de la morphologie. Des analyses plus fines, par exemple des dynamiques sémantiques que les différentes constructions mettent en place, ainsi que la prise en compte d'autre types de constructions (par exemple celles où l'élément spécifié est celui de gauche) nous permettront sans doute d'aboutir à un cadre plus complet.

\section{Références bibliographiques}

Ackema P., Neeleman A. (2010). The role of syntax and morphology in compounding. In Scalise \& Vogel (eds) (2010), 21-36.

Amiot, D. Dal, G. (2007). Integrating neoclassical combining forms into a lexeme-based morphology. In Booij et al. (eds) (2007), 323-336.

Amiot, D., Van Goethem, K. (2012). A constructional analysis of French -clé 'key' and Dutch sleutel- 'key' as in mot-clé / sleutelwoord 'key word'. Morphology, 22(3), 347-364.

Baeskow, H. (2004). Lexical Properties of Selected Non-native Morphemes of English. Tübingen : Narr.

Bauer, L. (1998). Is there a class of neoclassical compounds, and if so is it productive?. Linguistics, 36(3), 403-422.

Bisetto, A., Scalise, S. (2005). The classification of compounds. Lingue e linguaggio, IV(2), 319-332.

Booij, G. (2010a). Compound construction: Schemas or analogy? A construction morphology perspective. In Scalise \& Vogel (eds) (2010), 93-107.

Booij, G. (2010b). Construction Morphology. Oxford: Oxford University Press.

Booij, G., Ducceschi, L., Fradin, B., Guevara, E., Ralli, A., Scalise S. (eds). (2007). On-line Proceedings of the Fifth Mediterranean Morphology Meeting. Fréjus 15-18 September 2005. Bologna: Università di Bologna.

Corbin, D. (2001). Préfixes et suffixes : du sens aux catégories. Journal of French Language Studies, 11, 41-69.

Corbin, D., Plénat, M. (1992). Note sur l'haplologie des mots construits. Langue française, 96, 101-112.

Dal, G., Amiot D. (2008). Composition néoclassique et ordre des constituants. In : Amiot, D. (éd.), La composition dans les langues. Arras : Artois Presses Université, 89-113.

Fradin, B. (2000). Combining forms, blends and related phenomena. In Thornton A.M., Doleschal U. (eds), Extragrammatical and Marginal Morphology. München: Lincom Europa, 11-59. 
Fradin, B. (2009). IE, Romance: French. In : Lieber \& Štekauer (eds) (2009), 417-435.

Grand Robert: Grand Robert de la langue française [http://gr.bvdep.com/].

Hathout, N. (2011). Une approche topologique de la construction de mots. In Roché et al. (2011), 251-318.

Hathout, N., Montermini, F. (eds) (2007). Morphologie à Toulouse. Acte du colloque international de morphologie $4^{e}$ Décembrettes. München: Lincom Europa.

Iacobini, C. (2004). Composizione con elementi neoclassici. In Grossmann, M., Rainer, F. (eds), La formazione delle parole in italiano. Tübingen: Niemeyer, 69-95.

Koga, K. (2012). Enquête sur la productivité des noms composés du type pause-café en français. Mémoire de master. Université Michel de Montaigne - Bordeaux 3.

Lieber, R. Štekauer, P. (2009). Introduction: status and definition of compounding. In Lieber \& Štekauer (eds) (2009), 1-18

Lieber, R. Štekauer, P. (eds) (2009). The Oxford Handbook of compounding. Oxford: Oxford University Press.

Lüdeling, A., Schmid T., Kiokpasoglou, S. (2002). Neoclassical word formation in German. In Booij, G., van Maarle, J. (eds), Yearbook of Morphology 2001. Dordrecht: Foris, 253-283.

Namer, F. (2007). Composition néoclassique : est-on dans l'«hétéromorphosémie » ?. In Hathout \& Montermini (eds) (2007), 187-206

Namer, F., Villoing, F. (2007). Have cutthroats anything to do with tracheotomes? Distinctive properties of VN vs. NV compounds in French. In Booij et al. (eds) (2007), 105-124.

Noailly, M. (1990). Le substantif épithète. Paris : PUF.

Plag, I., (2003). Word Formation in English. Cambridge: Cambridge University Press.

Plénat, M. (1988). Morphologie des dérivés en -able. Cahiers de grammaire, 13, 101-132.

Plénat M. (2008). Le thème L de l'adjectif et du nom. In Durand, J., Habert, B., Laks, B. (eds), Congrès Mondial de Linguistique Française - CMLF ’08. Paris : Institut de Linguistique Française, 1613-1626.

Plénat, M. (2009). Les contraintes de taille. In Fradin, B., Kerleroux, F., Plénat, M. (eds), Aperçus de morphologie du français. Saint-Denis : Presses Universitaires de Vincennes, 47-63.

Plénat, M. (2011). Enquête sur différents effets des contraintes dissimilatives. In Roché et al. (2011), 145-190.

Ralli, A. (2007). Compound-marking in a cross-linguistic approach. In Hathout \& Montermini (eds) (2007), 207-220.

Ralli, A. (2013). Compounding in Modern Greek. Berlin : Springer.

Roché, M. (2009). Pour une morphologie lexicale. Mémoires de la Société de Linguistique de Paris. Nouvelle série $17,65-87$.

Roché (2010). Base, thème, radical. Recherches linguistiques de Vincennes, 39, 95-134.

Roché, M. (2011). Quelle morphologie ?. In Roche et al. (2011), 15-39.

Roché, M., Boyé, G., Hathout, N., Lignon, S., Plénat, M. (2011). Des unités morphologiques au lexique. Paris : Hermès Lavoisier.

Roché, M. Plénat, M. (2014). Le jeu des contraintes dans la sélection du thème présuffixal. Ce volume.

Scalise, S. (1990). Morfologia e lessico. Una prospettiva generativista. Bologna: Il Mulino.

Scalise, S., Vogel, I. (eds) (2010). Cross-Disciplinary Issues in Compounding. Amsterdam / Philadelphia: Benjamins.

TLFi. Trésor de la langue française informatisé [http://atilf.atilf.fr/].

Villoing, F. (2012). French compounds. Probus, 24 (1), 29-60. 
${ }^{1}$ Pour nous limiter au français, et sans vouloir forcément être exhaustifs, citons au moins les travaux de Amiot \& Dal (2007); Dal \& Amiot (2008); Fradin (2000); Namer (2007); Namer \& Villoing (2007); Villoing (2012).

${ }^{2}$ Plusieurs de ces exemples proviennent de Koga (2012). Nous remercions Kentaro Koga pour nous y avoir donné accès.

${ }^{3}$ Nous considérons, sans le discuter vraiment, que pisci ([pisi]) et lim ([lim]) sont des thèmes supplétifs, respectivement, des lexèmes POISSON et VASE.

${ }^{4}$ Les composés du grec en - $\varphi$ oß $\alpha$ présentent donc les mêmes problèmes d'analyse que les composés dits 'synthétiques' dans d'autres langues, comme par exemple l'anglais (cf. troublemaker 'fauteur de troubles', Ackema \& Neeleman 2010).

${ }^{5}$ À vrai dire, le $T L F i$ mentionne quelques mots formés au XIX ${ }^{\text {ème }}$ siècle dans lesquels le premier élément pouvait indiquer le sujet qui reçoit le traitement (anthropothérapie, zoothérapie), mais cet emploi ne nous semble plus disponible aujourd'hui.

${ }^{6}$ Cf. en particulier Scalise (1990: 121-124) ; Fradin (2000) ; Corbin (2001); Lüdeling et al. (2002) ; Plag (2003 : 155-158) ; Baeskow (2004) ; Iacobini (2004); Amiot \& Dal (2007); Villoing (2012); Ralli (2013).

${ }^{7}$ Nous utilisons cette notation pour indiquer la même séquence phonologique (modulo les modifications phonologiques régulières et la présence / absence d'une voyelle de liaison, cf. ci-dessous) qui peut apparaitre en première ou en deuxième position d'un mot complexe. Cela signifie, par exemple, que pour -metr- nous avons cherché dans le $T L F i$ les mots commençant par métr(o)- et ceux se terminant par -mètre.

${ }^{8}$ Amiot \& Dal (2007 : 332-333) affirment explicitement qu'en français il existe deux [log] : [log $]_{1}$, qui est le thème B («radical B ») d'un lexème (qu'elles n'indiquent pas, mais vraisemblablement MOT ou LANGAGE) et [log $]_{2}$, qui est l'exposant d'une régle de construction de noms de spécialistes.

${ }^{9}$ Un traitement plus complet devrait, bien entendu, tenir compte des lexèmes dans lesquels ces éléments sont suffixés et précédés d'un autre élément (néologisme, volumétrique), mais il est difficile, dans ces cas, de maintenir qu'ils sont simplement les bases d'un procédé de dérivation.

${ }^{10}$ Une propriété des composés néoclassiques qui est observée dans d'autres langues, et qui n'est pas pertinente en français, concerne, par exemple, leur comportement vis-à-vis de l'accent primaire de mot, qui, dans beaucoup de cas, rapproche encore davantage les éléments de composition finaux des 'véritables' suffixes (cf. Lüdeling et al., 2002 : 273-277 sur l'allemand ; Plag, 2003 : 156-157 sur l’anglais ; Iacobini, 2004 : 80 sur l’italien).

${ }^{11}$ Plus précisément, 41 de ces lexèmes sont recensés dans les dictionnaires (dont 20 uniquement dans le TLFi et 3 uniquement dans le Grand Robert) et 135 ont été obtenus par des recherches ciblées sur la Toile.

${ }^{12} \mathrm{http}: / /$ fr.wikipedia.org/wiki/Liste_de_langues. 